\title{
Type 1 Regulatory T-Cell
}

National Cancer Institute

\section{Source}

National Cancer Institute. Type 1 Regulatory T-Cell. NCI Thesaurus. Code C126755.

A population of CD4+, CD25-, and FoxP3- T-lymphocytes that are involved in immunotolerance. These cells secrete the immunosuppressive cytokines, tumor growth factor-beta (T GF-B) and interleukin-10, and may induce cell cycle arrest or apoptosis in effector T-cells. 